\section{Leonhard-Klein-Stiftung im Stifterverband für die Deutsche Wissenschaft e.V.}

Die Leonhard-Klein-Stiftung vergibt den

\section{Leonhard-Klein-Preis 2002 zur Förderung der Augen- chirurgie}

für innovative, wissenschaftliche Arbeiten auf dem Gebiet der mikrochirurgischen Instrumentenentwicklung und -anwendung sowie der mikrochirurgischen Operationstechnik, die über eine Modifikation bekannter Verfahren hinausgehen.

Der Preis ist mit

$15.000 €$

dotiert und kann sowohl einer Einzelpersönlichkeit als auch einer Forschergruppe zuerkannt werden. Er kann ausnahms- weise auch zu gleichen Teilen an zwei Preisträger verliehen werden. Die Preissumme soll vom Preisträger für die weitere Forschung auf dem Gebiet der Augenchirurgie verwendet werden.

Über die Vergabe des Preises entscheidet ein unter der Federführung des Vorstands der Deutschen Ophthalmologischen Gesellschaft zusammengesetztes Kuratorium.

Der Rechtsweg gegen die Entscheidung des Kuratoriums ist ausgeschlossen.

Es sind sowohl Eigenbewerbungen als auch Vorschläge Dritter möglich.

Die Arbeiten sind in deutscher oder englischer Sprache in fünffacher Ausfertigung bis zum 31. März 2002 zu richten an: Stifterverband für die Deutsche Wissenschaft e. V., z. Hd. Frau Dr. Marilen Macher, Postfach 1644 60, 45224 Essen.

\section{Literaturliste zum Thema Kontaktlinse}

(Stand Jänner 2002)

\section{Ein Service der Vereinigung kontaktlinsenanpassender Augenärzte}

Hier finden Sie eine aktuelle Liste von Literatur rund um die Kontaktlinse. Wir übernehmen keine Verantwortung für Richtigkeit der Angaben oder Lieferbarkeit.

Drei Bücher möchten wir besonders hervorheben:

1. Bürki: relativ leicht lesbar bringt der Autor Grundlegendes zur Kontaktologie. Viel Theorie, aber auch sehr guter Praxisbezug. Unentbehrlich.

2. Weinstock: die deutsche Ausgabe von Prof. Roth. Viele Illustrationen, auch ungewöhnliche Linsentypen werden behandelt.

3. Der ,neue“ Roth (erschienen 2002): Kontaktlinsenkomplikationen. Reich illustriert (die Fotos sind von ausgezeichneter Qualität), der Textteil ist übersichtlich und modern gegliedert, dieses Buch ist ein Muss (ausführliche Rezension im nächsten „Spektrum“)

Harald Schelle

Kontaktlinsen. Neues Sehen, selbst erleben

Trias, Stuttgart

$€ 17,95$

DIN-Normung

Kontaktlinsen. Normen, Richtlinien

Beuth-Verlag

$\in 59,31$
Frank J. Weinstock

Kontaktlinsen-Anpassung in Klinik und Praxis

Gustav-Fischer-Verlag

ISBN 3-437-00599-5

$€ 45,97$

Hans Walter Roth

Kontaktlinsen. Ein Ratgeber für den Patienten

Gustav-Fischer-Verlag

$€ 14,95$

Ernst Bürki

Augenärztliche Kontaktlinsenanpassung

Enke, Stuttgart

ISBN 3-432-99231-9

$€ 54,95$

Egon Faber, Joachim Rehm

Spaltlampenmikroskopie der Kontaktlinsenanpassung

Nieswand-Verlag, Kiel

$€ 59,90$

Hans Walter Roth

Kontaktlinsen-Komplikationen

Thieme, Stuttgart

$€ 99,00$

Gabriel Elie

Guide de Contactologie

Thieme, Stuttgart

$€ 54,95$

Autoren:

Dr. Claudia Wenk und Dr. Herbert Schuster 